RESEARCH H IGHLIGHTS

\title{
Down to the wire
}

Research just published in Nature reveals that hair-like protein structures - called pili - that are present on the surface of the soil bacterium Geobacter sulfurreducens not only help the microorganism attach to extracellular substrates - they also conduct electricity.

The ability of microorganisms to transfer electrons to external electron

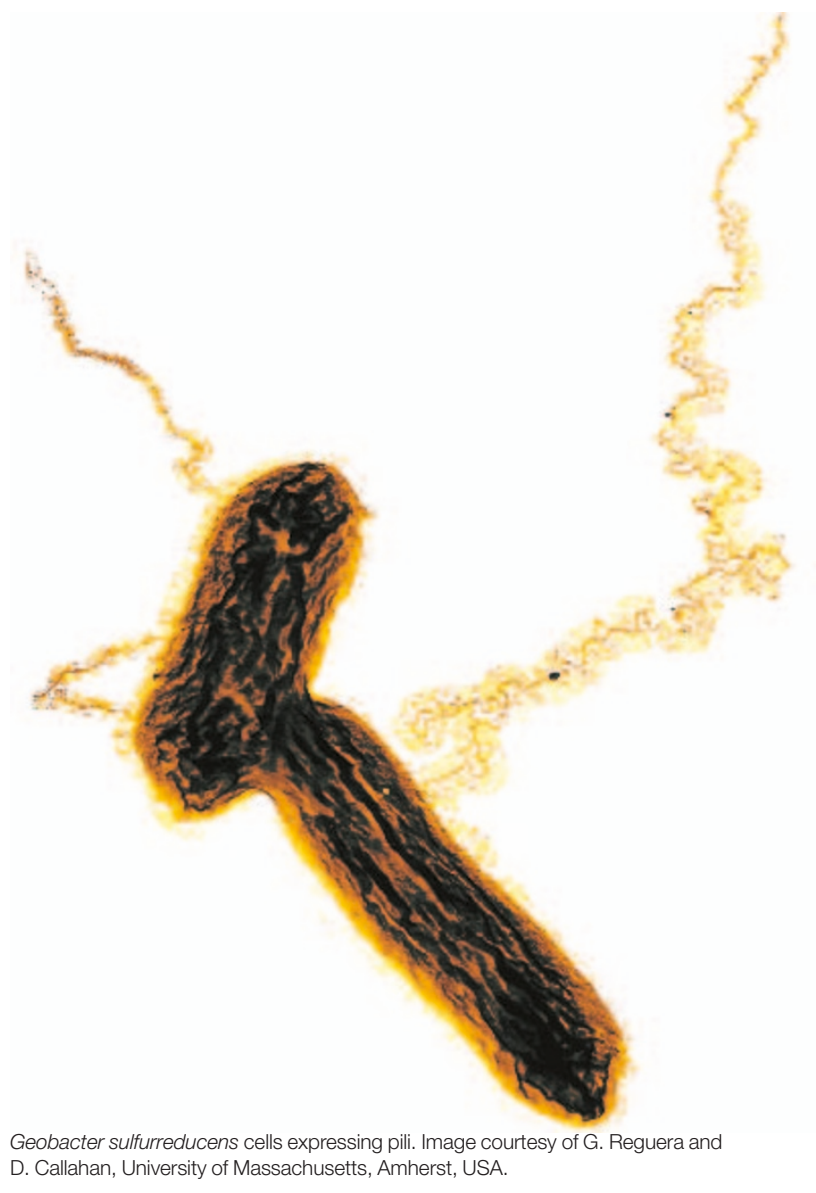

acceptors, such as $\mathrm{Fe}$ (III) oxides, is crucial to the biological processes that are involved in organic-matter breakdown and nutrient cycling within the soil environment. In many environments, including soil, Geobacter species are the predominant iron reducers, and previous work has shown that the bacteria must directly contact the $\mathrm{Fe}$ (III) oxides to reduce them. Up to now, the generally accepted mechanism of electron transfer in iron-reducing bacteria proposes that outer-membrane cytochromes are the agents that mediate transfer of electrons to Fe(III) oxide and that the pili structures on the surface of the microorganism are simply required for establishing contact with the surface of the oxides. To investigate this mechanism of electron transfer, Derek Lovley and colleagues generated a pilus-deficient mutant of $G$. sulfurreducens and assessed its ironreducing properties. Surprisingly, this analysis revealed that the mutant could no longer reduce Fe(III) oxides, yet maintained the ability to attach to their surface. Using conductingprobe atomic force microscopy to investigate the electrical properties of the pili, the authors also showed that these structures are highly conductive and could act as biological nanowires by providing an electrical connection between the bacterial cell and the surface of the Fe(III) oxides. As the pilus apparatus is anchored within the periplasm and outer membrane of the Gram-negative bacterium, Reguera et al. speculate that the pili accept electrons from electron-transfer proteins present in this location and that the structure then facilitates electron transfer to the extracellular Fe(III) oxide, completing the electrical circuit. The possibility that the pili might participate in other electron-transfer reactions will be the focus of future research.

As well as a potential new mechanism for bacterial electron transfer, these findings also raise the intriguing prospect of biologically produced nanowires being exploited as a 'next generation' conductive material with a multitude of possible applications in nanoelectronic devices.

David O'Connell

(2) References and links ORIGINAL RESEARCH PAPER Reguera, G. et al. Extracellular electron transfer via microbial nanowires. Nature 435, 1098-1101 (2005) FURTHER READING Lovley, D. R. Cleaning up with genomics: applying molecular biology to bioremediation. Nature Rev. Microbiol. 1, 35-44 (2003) 